\title{
Courant 代数胚与广义复几何
}

献给钱敏教授 90 华诞

\author{
陈酌 ${ }^{1}$, 刘张炬 ${ }^{2 *}$, 徐平 ${ }^{3}$ \\ 1. 清华大学数学科学系, 北京 100084 ; \\ 2. 北京大学数学科学学院, 北京 100871 ; \\ 3. Department of Mathematics, The Pennsylvania State University, University Park 16802, USA \\ E-mail: zchen@math.tsinghua.edu.cn, liuzj@pku.edu.cn, ping@math.psu.edu
}

收稿日期: 2017-07-17；接受日期: 2017-08-16；网络出版日期: 2017-10-19; * 通信作者

国家自然科学基金 (批准号: 11471179 和 11471139) 资助项目

\begin{abstract}
摘要 本文综述 Courant 代数胚的研究背景、发展历史、重要理论及其应用. 本文回顾和介绍该领域 中一些关键的发现和进展, 并重点阐述 Courant 代数肧定义的来龙去脉, 以及相应的 Dirac 结构、李 双代数胚、Clifford 构造、旋量表示、广义复几何以及正则 Courant 代数胚的理论框架和一些主要的 结论.
\end{abstract}

关键词 Courant 代数胚 Dirac 结构 李双代数胚 广义复几何

MSC (2010) 主题分类 $53 \mathrm{C} 15,53 \mathrm{D} 17,57 \mathrm{R} 15,58 \mathrm{~A} 10,15 \mathrm{~A} 66,17 \mathrm{~B} 63,17 \mathrm{~B} 66$

\section{1 引言}

Courant 代数胚是由 Weinstein 与本文的两位作者刘张炬和徐平在文献 [1] 中引进的一个新的数 学结构, 当时的目的比较单纯, 就是要寻找一个适当的数学结构来刻画李双代数胚偶 (double). 随着 时间的发展, 人们发现 Courant 代数胚不但自身具有十分丰富的数学性质, 而且在微分几何, 特别是 广义复几何、L- 无穷代数、gerbe 以及拓扑量子场论 (TQFT)、双场理论 (double field theory) 和弦论 (string theory) 等若干数学与物理领域有广泛的应用. Courant 代数肧和 Dirac 结构理论自身在不断的 发展, 亦在力学和控制论领域也发现广泛的应用. 例如, 端口 Hamilton 系统、隐 Hamilton 系统、AKSZ sigma 模型和超引力等 (参见文献 [2-6]). 有关 Courant 代数胚以及相关问题引起了一批数学家和物 理学家的关注, 并且成为了当前活跃的研究课题之一.

首先就 Courant 代数胚的产生背景与发展进程作一个简单的回顾. Courant 代数胚的概念来自于 与物理密切相关的两个数学领域: Poisson 几何和量子群. 陈省身先生的门生 Weinstein 于 1988 年引 进了 Poisson 几何中的一个重要的概念一Poisson 群肧 ${ }^{[7]}$, 即李群胚上配置一个可乘的 Poisson 张量. 几年之后, 作为 Poisson 群胚的无穷小对应构造, Machenzie 和 $\mathrm{Xu}{ }^{[8]}$ 给出了李双代数胚 $\left(A, A^{*}\right)$ 的定 义, 其中 Poisson 流形的切丛和余切丛 $\left(T M, T^{*} M\right)$ 成为一个典型的例子. 上述的 Poisson 群胚和李双 
代数胚实际上分别是 Poisson 李群和李双代数的推广, 二者来自于著名的量子群的半经典极限 (参见 文献 [9]). 对李双代数 $\left(\mathfrak{g}, \mathfrak{g}^{*}\right)$, Drinfel'd ${ }^{[10]}$ 引进了李双代数偶的概念. 他证明了在直和空间 $D=\mathfrak{g} \oplus \mathfrak{g}^{*}$ 上存在唯一的李代数结构满足由配对定义的非退化二次型是不变内积, 并且 $\mathfrak{g}$ 和 $\mathfrak{g}^{*}$ 同为极大迷向子 代数. 进一步地, Drinfel'd ${ }^{[10]}$ 利用 Manin 三元组对李双代数结构与分类进行了深入的研究.

为了运用 Manin 三元组研究李双代数胚, 首先要引入李双代数胚偶的概念. 由于一些几何上的 障碍, 发现李双代数胚偶的定义比预想的要复杂, 这就促使了一个新的数学结构 Courant 代数胚的引 进 (参见文献 [1]). 以 “Courant” 命名这个结构源于微分几何中 Courant 括号. Courant 为了研究流 形上的 Dirac 结构, 在直和向量丛 $T M \oplus T^{*} M$ 的截面空间上定义了一个后来以他的名字命名的括号. 这个括号的奇异之处在于其本身不满足 Jacobi 恒等式, 但是 Dirac 结构, 即一类极大可积子丛成为李 代数胚, 包括预辛结构、Poisson 结构和叶状结构等人们所熟知的几何结构 (参见文献 [11]). 另外, 在 Cabras 和 Vinogradov 的工作 [12] 中, 也有完全一致的构造.

现在人们称直和向量从 $T M \oplus T^{*} M$ 上由 Courant 括号所定义的 Courant 代数胚为标准 Courant 代数胚或者称之为微分流形 $M$ 的广义切丛. Severa 证明了流形上标准 Courant 代数胚可以通过一 个闭的三阶微分形式 $\omega$ 进行形变, 得到一个恰当的 Courant 代数胚. 称 $\omega$ 的同调类为 Severa 类 ${ }^{[13]}$. Severa 类有明确的物理意义, 在弦论中为 Neveu-Schwarz 通量.

正是以广义切从为起点, 取 $T M \oplus T^{*} M$ 的复化, Hitchin ${ }^{[14]}$ 和他的牛津学派, 以及一批数学家和 物理家建立和发展了 “广义几何” [15], 该理论已经成为了一个丰富多彩的数学理论并且广泛应用于其 他数学和物理领域的研究. 例如, 复几何与辛几何在广义复几何的框架中得到了统一, 由此发现了这 两种几何之间有许多相通之处, 于是人们对广义复几何寄予希望去揭示隐藏在几何和物理中的一个核 心问题 “镜像对称”之中的秘密 (参见文献 [16]). 另外, 在双场理论及 MIT 学派的很多研究工作中, Courant 代数胚已经成为标准的工具之一.

由于局限在李理论框架下工作, Courant 代数胚的原始定义中的括号运算是反对称的. 这个括号 满足的条件看上去有些怪异、反常. 例如, 其不满足 Jacobi 恒等式. 于是, Liu 等 ${ }^{[1]}$ 就对这个括号进行 了修正. 修正后的括号不再满足反对称条件, 但是具有若干好的性质. 这个非反对称的括号当时对我 们来说是非常神秘的, 在本文中也作为一个公开问题提出来了.

不久这个问题就得到了澄清. Kosmann-Schwarzbach、Severa 和徐平等先后发现了原来此神秘的 非反对称括号满足 Leibniz 条件. 上述几个人的相关的工作都没有正式发表, Roytenberg 将结论写入了 他的博士论文 [17], 并与 Weinstein 一起将其发表在文献 [18]. 这类代数结构是法国数学家 Loday [19] 作为非交换李代数引进的, 因此, Leibniz 代数也被称为 Loday 代数. 另外, 由于已故俄国女数学家 Dorfman ${ }^{[20]}$ 为了研究无穷维可积 Hamilton 系统, 也利用类似括号建立了 Dirac 结构的代数理论, 现 在这个括号通常被称为 “Dorfman 括号” 以及更一般的 Courant-Dorfman 括号. 我们指出, Cabras 和 Vinogradov 在他们的工作 [12] 中, 也有完全相同的构造.

Courant 代数胚可以看成一类配有不变内积的 Leibniz 代数的几何实现. 事实上, 早在 2001 年, 在 Alekseev 和 Xu 的一篇未正式发表的论文 [21] 中, 已经发现了构造 Courant 代数胚的 Clifford 实现方 式, 此即最早的导出括号方法 (derived brackets). 同时, Weinstein、Severa 和 Roytenberg 又利用分次 Poisson 流形的极小辛实现、同调 Hamilton 向量场和辛超流形定义了 Courant 代数胚的导出括号. 该 结果后来写入了 Roytenberg 的博士论文 [17] 中. 实际上, 导出括号方法是李理论和 Poisson 几何的一 个基本方法, 感兴趣的读者可以参见 Kosmann-Schwarzbach 的综述 [22], 特别是其中对标准 Courant 代数胚 $T M \oplus T^{*} M$ 上的 Courant 括号定义的导出括号的详细论述.

Roytenberg 在他的博士论文中还发现了 Courant 代数胚原始的反对称括号实际上形成了一个李 -2 
代数、即只有两项的 $L$ - 无穷代数, 同时也是李代数的一种范畴化. 通过范畴化得到的结构称为高阶结 构, 由此可知 Courant 代数胚本质上是一种高阶结构 ${ }^{[18]}$, 当初显现出来的一些怪异和反常的性质也就 不足为奇了. 高阶结构相关数学与物理领域的应用可以参见文献 [23].

我们特别介绍关于 Courant 代数胚同调论的重要进展: Severa ${ }^{[24]}$ 最早发现了关于正合 Courant 代数胚的分类定理. Roytenberg ${ }^{[17]}$ 首先提出了标准同调, 这种同调论计算非常困难. 后来, Stiénon 和 $\mathrm{Xu}{ }^{[25]}$ 提出了初级 (naive) 同调, 并提出了这样的猜想: 如果 Courant 代数胚是正则的, 那么标准同调 与初级同调同构. 该猜想已经被 Ginot 和 Grutzmann ${ }^{[26]}$ 证明.

对称性是数学和物理学的基本特性, 随着时间的推移, 人们发现用李代数和李群来刻画对称性有 很大的局限性. 李代数胚和李群胚为深入理解和研究对称性提供了更广阔的理论框架, 可以参见文 献 [27]. Courant 代数胚则为研究高阶数学结构的高阶对称性提供了有力的工具.

正则 Courant 代数胚与规范场论密切相关, 特别是传递的 Courant 代数胚基本上可以视为主纤维 从的 Atiyah 代数胚的余切扩张. Chen 等 ${ }^{[28]}$ 对正则 Courant 代数胚的结构与分类进行了深入的研究, 指出了 Pontryagin 类是构造正则 Courant 代数胚的一个障碍. 当 Pontryagin 类非平凡时, Liu 等 [29] 定义了扭 Courant 代数胚从而得到了 Leibniz 2- 代数的几何实现. 受 Weinstein 的 Omni-Lie 代数的 启发, Chen 等 ${ }^{[30]}$ 定义了 $E$-Courant 代数胚, 其中 $E$ 是一个向量丛. 这个结构统一了目前存在的若 干个 Courant 代数胚的变形, 包括 AV-Courant 代数胚、共形 Courant 代数胚、高阶 Courant 代数 胚、Courant-Jacobi 代数胚和 Omni-Lie 代数胚等结构. 另外, 近年来关于 Courant 代数胚的积分有广 泛的研究, 读者可参见文献 [31-33].

目前 Courant 代数胚的理论与应用还处于发展阶段, 有许多内容都不能包含在这篇简要的综述 之中, 这里仅介绍了一些 Courant 代数胚的基本内容. 希望读者对这个领域有一个初步认识, 进一步 可以参见文献 [34]. 本文各节主要内容安排如下: 第 2 节介绍一些预备知识: Poisson 李群、李双代 数、Manin 三元组、李代数胚与李双代数胚、Courant 括号和 Dirac 结构; 第 3 节介绍 Courant 代数 胚的定义、李双代数胚与 Courant 代数胚的关系、Severa 类, 以及用于刻画形变的 Maurer-Cartan 方 程; 第 4 节讲述 Courant 代数胚的 Clifford 实现和导出括号; 第 5 节介绍广义复几何; 第 6 节讨论正 则 Courant 代数胚的结构与分类.

\section{2 预备知识}

\subsection{Poisson 李群、李双代数和 Manin 三元组}

Poisson 李群是 Poisson 流形和李群的结合体:

定义 1 称一个李群 $G$ 为 Poisson 李群, 如果 $G$ 上有 Poisson 张量 $\pi$, 使得 $G$ 的群乘法运算 $\mu: G \times G \rightarrow G, \mu\left(g_{1}, g_{2}\right)=g_{1} g_{2}$ 是 Poisson 映射.

上述条件的显式表达为

$$
\left\{f_{1}, f_{2}\right\}\left(g g^{\prime}\right)=\left\{f_{1} \circ L_{g}, f_{2} \circ L_{g}\right\}\left(g^{\prime}\right)+\left\{f_{1} \circ R_{g^{\prime}}, f_{2} \circ R_{g^{\prime}}\right\}(g),
$$

其中 $f_{1}$ 和 $f_{2}$ 是 $G$ 上的光滑函数, $g$ 和 $g^{\prime}$ 是群中的元素. 另一种等价的条件表达为

$$
\pi\left(g g^{\prime}\right)=L_{g *}\left(\pi\left(g^{\prime}\right)\right)+R_{g^{\prime *}}(\pi(g)) .
$$

关于李双代数, 主要追溯到 Drinfeld 在量子群及其经典极限即上述 Poisson 李群方面的研究工作 (参见文献 $[9,10]$ ). 
定义 2 设 $\mathfrak{g}$ 及其对偶空间 $\mathfrak{g}^{*}$ 都是李代数, 如果它们的直和空间 $D=\mathfrak{g} \oplus \mathfrak{g}^{*}$ 也构成李代数, 并 且 $D$ 的李括号由原来 $\mathfrak{g}$ 和 $\mathfrak{g}^{*}$ 的李括号及它们互相之间的余伴随作用生成, 则称 $\left(\mathfrak{g}, \mathfrak{g}^{*}\right)$ 是李双代数. 可以证明, 若 $(G, \pi)$ 是 Poisson 李群, 则 $G$ 的切李代数是李双代数.

特别是在文献 [10] 中, Drinfeld 对一个李双代数 $\left(\mathfrak{g}, \mathfrak{g}^{*}\right)$, 考虑了所谓的二重化 (double): $\mathfrak{g} \oplus \mathfrak{g}^{*}$. 在 $\mathfrak{g} \oplus \mathfrak{g}^{*}$ 上, 有标准的非退化不变双线性型, 以及李代数结构, 且限制到 $\mathfrak{g}$ 和 $\mathfrak{g}^{*}$ 上就是原有的李代数. 这样的结构如今称为 Manin 三元组:

定义 3 设李代数 $\mathfrak{d}$ 是二次李代数, 即存在 $\mathfrak{d}$ 上的非退化二次型 $\langle\cdot, \cdot\rangle$, 且满足

$$
\langle[x, y], z\rangle=-\langle y,[x, z]\rangle, \quad \forall x, y, z \in \mathfrak{d} .
$$

如果 $\mathfrak{d}$ 中存在两个迷向子代数 $\mathfrak{g}$ 和 $\mathfrak{h}$ (即 $\langle\cdot, \cdot\rangle$ 限制在 $\mathfrak{g} \times \mathfrak{g}, \mathfrak{h} \times \mathfrak{h}$ 都为零), 使得 $\mathfrak{d}=\mathfrak{g} \oplus \mathfrak{h}$, 则称 $(\mathfrak{d}, \mathfrak{g}, \mathfrak{h})$ 构成 Manin 三元组.

容易看出, Manin 三元组中的 $(\mathfrak{g}, \mathfrak{h})$ 互为对偶空间: $\mathfrak{h} \cong \mathfrak{g}^{*}$. 事实上可以证明这一对李代数恰好构 成李双代数.

\section{2 李代数胚与李双代数胚}

李群胚 ${ }^{[35]}$ 和李代数胚 ${ }^{[36]}$ 也是 Poisson 几何中和数学物理研究中常见的数学对象, 感兴趣的读者 请参阅系统介绍这方面知识的文献 [37]. 我们简要回顾李代数胚的定义:

定义 4 (李代数胚) 设 $A$ 是流形 $M$ 上的向量从 (某个特征为 0 的数域 $\mathbb{K}$ 上的向量从, 通常默 认为 $\mathbb{R}$ 或 $\mathbb{C}$ ). 如果 $A$ 的截面空间 $\Gamma(A)$ 上存在一个李代数结构 $[\cdot, \cdot]_{A}$, 并且存在一个 $M$ 上的丛同态 $\rho_{A}: A \rightarrow T_{\mathbb{K}} M$ (这里 $T_{\mathbb{K}} M=T M \otimes \mathbb{K}$ ) 满足

(1) $\rho_{A}$ 诱导了一个李代数同态: $\rho_{A}: \Gamma(A) \rightarrow \Gamma\left(T_{\mathbb{K}} M\right)$, 即对任意的 $X, Y \in \Gamma(A)$, 都有

$$
\rho_{A}\left([X, Y]_{A}\right)=\left[\rho_{A}(X), \rho_{A}(Y)\right]
$$

(2) 如下的 Leibniz 恒等式成立:

$$
[X, f Y]_{A}=f[X, Y]_{A}+\left(\rho_{A}(X) f\right) Y, \quad \forall X, Y \in \Gamma(A), \quad f \in C^{\infty}(M, \mathbb{K}),
$$

那么称 $A$ 是 $M$ 上的李代数胚, 并称 $\rho_{A}$ 为李代数胚的针 (anchor).

在实际应用中, 我们通常把上述李代数胚结构拓展成为外代数 $\Gamma\left(\wedge^{\bullet} A\right)$ 上的 Schouten 括号, 依然 记作 $[\cdot, \cdot]_{A}$.

定义 5 (Schouten 括号) 假设 $A$ 是 $M$ 上的李代数胚. 对任意的 $X_{1} \wedge \cdots \wedge X_{p} \in \Gamma\left(\wedge^{p} A\right)$ 和 $Y_{1} \wedge \cdots \wedge Y_{q} \in \Gamma\left(\wedge^{q} A\right)$, 其 Schouten 括号定义为

$$
\left[X_{1} \wedge \cdots \wedge X_{p}, Y_{1} \wedge \cdots \wedge Y_{q}\right]_{A}=\sum_{i, j}(-1)^{i+j}\left[X_{i}, Y_{j}\right]_{A} \wedge X_{1} \wedge \cdots \hat{X}_{i} \cdots \wedge X_{p} \wedge Y_{1} \wedge \cdots \hat{Y}_{j} \cdots \wedge Y_{q}
$$

特别地, 对任意的 $X \in \Gamma(A)$ 和 $f \in C^{\infty}(M, \mathbb{K})$, 其 Schouten 括号定义为

$$
[X, f]_{A}=-[f, X]_{A}=\rho_{A}(X) f
$$

其中 $\rho_{A}$ 为李代数胚 $A$ 的针. 
设 $A$ 是李代数胚, 则在 $\Gamma\left(\wedge^{\bullet} A^{*}\right)$ 上, 有诱导的外微分运算 $d_{A}: \Gamma\left(\wedge^{\bullet} A^{*}\right) \rightarrow \Gamma\left(\wedge^{\bullet} A^{*}\right)$ 满足如下导 子条件:

$$
d_{A}(\omega \wedge \eta)=d_{A} \omega \wedge \eta+(-1)^{|\omega|} \omega \wedge d_{A} \eta, \quad \forall \omega, \eta \in \Gamma\left(\wedge^{\cdot} A^{*}\right)
$$

其具体定义如下.

定义 6 (李代数胚微分) 李代数胚 $A$ 上的微分 $d_{A}: \Gamma\left(\wedge^{k} A^{*}\right) \rightarrow \Gamma\left(\wedge^{k+1} A^{*}\right)$ 定义为

$$
\begin{aligned}
d_{A} \sigma\left(X_{0}, \ldots, X_{k}\right)= & \sum_{i}(-1)^{i} \rho_{A}\left(X_{i}\right) \sigma\left(X_{0}, \ldots, \hat{X}_{i}, \ldots, X_{k}\right) \\
& +\sum_{i<j}(-1)^{i+j} \sigma\left(\left[X_{i}, X_{j}\right]_{A}, X_{0}, \ldots, \hat{X}_{i}, \ldots, \hat{X}_{j}, \ldots, X_{k}\right),
\end{aligned}
$$

其中 $\sigma \in \Gamma\left(\wedge^{k} A^{*}\right), X_{i} \in \Gamma(A), \rho_{A}$ 是李代数胚 $A$ 的针, $A^{*}$ 是 $A$ 的对偶丛.

特别地,

$$
\begin{aligned}
& d_{A} f=\rho_{A}^{*}(d f), \quad \forall f \in C^{\infty}(M, \mathbb{K}), \\
& \left\langle d_{A} \xi, a_{1} \wedge a_{2}\right\rangle=\rho_{A}\left(a_{1}\right)\left\langle\xi, a_{2}\right\rangle-\rho_{A}\left(a_{2}\right)\left\langle\xi, a_{1}\right\rangle-\left\langle\xi,\left[a_{1}, a_{2}\right]_{A}\right\rangle, \quad \forall \xi \in \Gamma\left(A^{*}\right), \quad a_{1}, a_{2} \in \Gamma(A) .
\end{aligned}
$$

注 7 由于 $[\cdot, \cdot]_{A}$ 满足 Jacobi 恒等式, 容易验证 $d_{A}$ 满足 $d_{A}^{2}=0$. 因此, $\left(\Gamma\left(\wedge A^{*}\right), d_{A}\right)$ 是一个微 分上链复形 (differential cochain complex). 相应的同调称为李代数胚 $A$ 的上同调环, 记作 $H^{\bullet}(A)$.

定义 8 若 $A$ 是李代数胚, 且其对偶丛 $A^{*}$ 也是李代数胚, 如果下面的条件成立, 则称 $A$ 与 $A^{*}$ 构成李双代数胚:

$$
d_{A}[\omega, \eta]_{*}=\left[d_{A} \omega, \eta\right]_{*}+(-1)^{|\omega|-1}\left[\omega, d_{A} \eta\right]_{*}, \quad \forall \omega, \eta \in \Gamma\left(\wedge^{\bullet} A^{*}\right),
$$

其中 $[\cdot, \cdot]_{*}$ 是由 $A^{*}$ 的李代数胚结构所扩展的 Schouten 括号. 换言之, $\left(\Gamma\left(\wedge^{\bullet} A^{*}\right),[\cdot, \cdot]_{*}, d_{A}\right)$ 构成微分 Gerstenhaber 代数 ${ }^{[38]}$.

可以证明, 李双代数胚的定义是对称的.

定理 $9^{[8]}$ 若 $A$ 与 $A^{*}$ 构成李双代数胚, 则 $A^{*}$ 与 $A$ 也构成李双代数胚. 反之亦然.

我们列举几个简单的例子. 前述李双代数 $\left(\mathfrak{g}, \mathfrak{g}^{*}\right)$ 显然是特殊的李双代数胚; 给定 Poisson 流形 $M$, $T^{*} M$ 构成李代数胚, 且 $\left(T M, T^{*} M\right)$ 构成 (实) 李双代数胚; 给定流形 $M$ 上的广义复结构, 则其两个 共轭 Dirac 结构 $L$ 与 $\bar{L}$ 构成 (复) 李双代数胚, 详见第 5 节.

\subsection{Courant 括号与 Dirac 结构}

Courant 括号是微分流形上向量场的括号和李导数的自然推广. 设 $M$ 是光滑流形, 记

$$
E=\left(T M \oplus T^{*} M\right) \otimes \mathbb{K}=T_{\mathbb{K}} M \oplus T_{\mathbb{K}}^{*} M,
$$

称作 $M$ 的广义切丛. 在 $\Gamma(E)$ 上定义下面的括号运算:

$$
\left[X_{1}+\xi_{1}, X_{2}+\xi_{2}\right]=\left[X_{1}, X_{2}\right]+\left(L_{X_{1}} \xi_{2}-L_{X_{2}} \xi_{1}\right)-\frac{1}{2} d\left(i_{X_{1}} \xi_{2}-i_{X_{2}} \xi_{1}\right),
$$

其中 $X_{1}, X_{2} \in \mathfrak{X}_{\mathbb{K}}(M), \xi_{1}, \xi_{2} \in \Omega_{\mathbb{K}}(M)$. 此括号运算称为 Courant 括号, 这是 Courant 最早在文献 [11] 引入的. 
事实上, 在实际应用中, 人们常常使用非反对称形式的运算 (亦称 Dorfman 括号)

$$
\left(X_{1}+\xi_{1}\right) \circ\left(X_{2}+\xi_{2}\right)=\left[X_{1}, X_{2}\right]+\left(L_{X_{1}} \xi_{2}-i_{X_{2}} d \xi_{1}\right) .
$$

其反对称化恰好就是上述 Courant 括号.

另外, 在向量从 $E$ 上定义一个非退化的双线性二次型, 简称标准内积:

$$
\left\langle X_{1}+\xi_{1}, X_{2}+\xi_{2}\right\rangle=\frac{1}{2}\left(i_{X_{1}} \xi_{2}+i_{X_{2}} \xi_{1}\right) .
$$

在上述 Courant 括号和标准内积下, 有如下定义:

定义 10 一个流形 $M$ 上的 Dirac 结构, 如 $D$, 指的是 $E$ 的一个相对于标准内积的极大迷向子 从 $D \subset E$ (迷向即 $\langle D, D\rangle=0$ ), 且满足如下的封闭性 (又称可积性或对合性 (involutive)) 条件:

$$
[\Gamma(D), \Gamma(D)] \subset \Gamma(D) .
$$

典型的 Dirac 结构包括 Poisson 结构、预辛结构和可积分布等. 它们对应的 Dirac 结构的详细方 法参见文献 [11]. 本文后面提到的广义复结构也是一种 Dirac 结构.

\section{Courant 代数胚}

\subsection{Courant 代数胚的定义}

首先简单介绍 Courant 代数胚的公理化定义, 这个概念是 $\mathrm{Liu}$ 等 ${ }^{[1]}$ 引入的, 简单地讲, 就是把李 双代数胚 (详见第 3 节)、Courant、Dorfman 和 Vinogradov 在 $T M \oplus T^{*} M$ (这里 $M$ 是光滑流形) 上 引入的代数结构综合在一起的数学对象.

一个 Courant 代数胚由下列要素构成: 向量从 $E$ 、逐纤维定义的 $E$ 上的非退化二次型 $\langle\cdot, \cdot\rangle$ 、针 映射 (anchor) $\rho$, 以及一个被称作 Courant 括号 (或 Dorfman 括号) 的运算 $[\cdot, \cdot]$ (或 $\cdot 0 \cdot$ ). 这些要素要 满足一组比较复杂的相容性条件, 详见下面的两种等价定义方式:

定义 11 (Courant 代数胚) 设 $E$ 是 $M$ 上的 (以 $\mathbb{K}$ 为系数域的) 向量丛. 如果 $M$ 的光滑截面空 间 $\Gamma(E)$ 上存在一个非退化的对称双线性型 $\langle\cdot, \cdot\rangle$ (简称内积) 和一个反对称的括号 $[\cdot, \cdot]$ (称作 Courant 括号), 以及一个丛同态 $\rho: E \rightarrow T_{\mathbb{K}} M$, 使得下述条件成立:

(i) $\rho\left(\left[e_{1}, e_{2}\right]\right)=\left[\rho\left(e_{1}\right), \rho\left(e_{2}\right)\right], \forall e_{1}, e_{2} \in \Gamma(E)$;

(ii) $\operatorname{Jac}\left(e_{1}, e_{2}, e_{3}\right)=\mathcal{D}\left(\operatorname{Nij}\left(e_{1}, e_{2}, e_{3}\right)\right), \forall e_{1}, e_{2}, e_{3} \in \Gamma(E)$;

(iii) $\left[e_{1}, f e_{2}\right]=f\left[e_{1}, e_{2}\right]+\left(\rho\left(e_{1}\right) f\right) e_{2}-\left\langle e_{1}, e_{2}\right\rangle \mathcal{D} f, \forall e_{1}, e_{2} \in \Gamma(E), f \in \mathrm{C}^{\infty}(M, \mathbb{K})$;

(iv) $\rho \circ \mathcal{D}=0$, 即 $\langle\mathcal{D} f, \mathcal{D} g\rangle=0, \forall f, g \in \mathrm{C}^{\infty}(M, \mathbb{K})$;

(v) $\rho(e)\left\langle h_{1}, h_{2}\right\rangle=\left\langle\left[e, h_{1}\right]+\mathcal{D}\left\langle e, h_{1}\right\rangle, h_{2}\right\rangle+\left\langle h_{1},\left[e, h_{2}\right]+\mathcal{D}\left\langle e, h_{2}\right\rangle\right\rangle, \forall e, h_{1}, h_{2} \in \Gamma(E)$,

其中 $\mathrm{Jac}$ 和 $\mathrm{Nij}$ 算子的定义如下: 对任意 $A, B, C \in \Gamma(E)$, 有

$$
\operatorname{Jac}(A, B, C)=[[A, B], C]+[[B, C], A]+[[C, A], B]
$$

和

$$
\operatorname{Nij}(A, B, C)=\frac{1}{3}(\langle[A, B], C\rangle+\langle[B, C], A\rangle+\langle[C, A], B\rangle) .
$$

算符 $\mathcal{D}: \mathrm{C}^{\infty}(M, \mathbb{K}) \rightarrow \Gamma(E)$ 由

$$
\langle\mathcal{D} f, e\rangle=\frac{1}{2} \rho(e) f, \quad \forall e \in \Gamma(E), \quad f \in \mathrm{C}^{\infty}(M, \mathbb{K})
$$


给出. 赋予这些结构的向量丛 $E$ 是 $M$ 上的一个 Courant 代数胚.

定义 12 (Courant 代数胚等价定义) 称一个向量丛 $E \rightarrow M$ 为 Courant 代数胚, 如果存在逐 纤维定义的内积 $\langle\cdot, \cdot\rangle$, 以及丛映射 $\rho: E \rightarrow T_{\mathbb{K}} M$ (称作针) 和 $\Gamma(E)$ 上的 $\mathbb{K}-$ 双线性运算 $\cdot \circ$. (称作 Dorfman 括号), 使得对任何 $f \in C^{\infty}(M, \mathbb{K}), e_{1}, e_{2}, e_{3} \in \Gamma(E)$, 下列条件成立:

$$
\begin{aligned}
& e_{1} \circ\left(e_{2} \circ e_{3}\right)=\left(e_{1} \circ e_{2}\right) \circ e_{3}+e_{2} \circ\left(e_{1} \circ e_{3}\right), \\
& \rho\left(e_{1} \circ e_{2}\right)=\left[\rho\left(e_{1}\right), \rho\left(e_{2}\right)\right], \\
& e_{1} \circ\left(f e_{2}\right)=\left(\rho\left(e_{1}\right) f\right) e_{2}+f\left(e_{1} \circ e_{2}\right), \\
& \frac{1}{2}\left(e_{1} \circ e_{2}+e_{2} \circ e_{1}\right)=\mathcal{D}\left\langle e_{1}, e_{2}\right\rangle, \\
& \mathcal{D} f \circ e_{1}=0, \\
& \rho\left(e_{1}\right)\left\langle e_{2}, e_{3}\right\rangle=\left\langle e_{1} \circ e_{2}, e_{3}\right\rangle+\left\langle e_{2}, e_{1} \circ e_{3}\right\rangle,
\end{aligned}
$$

其中 $\mathcal{D}: C^{\infty}(M, \mathbb{K}) \rightarrow \Gamma(E)$ 是 $\mathbb{K}$ - 线性映射, 定义为

$$
\langle\mathcal{D} f, e\rangle=\frac{1}{2} \rho(e) f .
$$

注意到 (3.4) 给出了 Dorfman 括号的反对称化. 我们指出这两种定义的关联在于

$$
\left[e_{1}, e_{2}\right]=\frac{1}{2}\left(e_{1} \circ e_{2}-e_{2} \circ e_{1}\right),
$$

也就说 Dorfman 括号的反对称化就是 Courant 括号. 反过来, 有

$$
e_{1} \circ e_{2}=\left[e_{1}, e_{2}\right]+\mathcal{D}\left\langle e_{1}, e_{2}\right\rangle .
$$

定义 13 设 $E$ 是如上定义的 Courant 代数胚, 称一个 $E$ 的子丛 $D \subset E$ 是 Dirac 结构, 如果

(1) $D$ 关于内积 $\langle\cdot, \cdot\rangle$ 是极大迷向的, 即 $\langle D, D\rangle=0$, 且对任意 $X \in E,\langle X+D, X+D\rangle=0$ 推出 $X \in D$

(2) $D$ 的截面空间关于 Courant 括号 (或者 Dorfman 括号) 封闭: $[\Gamma(D), \Gamma(D)] \subset \Gamma(D)$.

推论 14 继承 $E$ 的括号运算和针映射, Dirac 结构 $D$ 拥有自然的李代数胚结构.

\subsection{Courant 代数胚和李双代数胚}

下面的两个定理阐述了李双代数胚与 Courant 代数胚的关系.

定理 $15^{[1]}$ 若 $A$ 与 $A^{*}$ 构成李双代数胚, 则 $E=A \oplus A^{*}$ 拥有 Courant 代数胚结构.

(1) 标准对称型, 即内积, 定义为

$$
\langle X+\xi, Y+\eta\rangle:=\frac{1}{2}(\xi(Y)+\eta(X)) ;
$$

(2) 针映射定义为

$$
\rho(X+\xi)=\rho_{A}(X)+\rho_{*}(\xi) ;
$$

(3) Courant 括号定义为

$$
\left[e_{1}, e_{2}\right]=\left[X_{1}+\xi_{1}, X_{2}+\xi_{2}\right]
$$




$$
\begin{aligned}
= & \left(\left[X_{1}, X_{2}\right]_{A}+L_{\xi_{1}} X_{2}-L_{\xi_{2}} X_{1}-d_{*}\left(e_{1}, e_{2}\right)_{-}\right) \\
& +\left(\left[\xi_{1}, \xi_{2}\right]_{*}+L_{X_{1}} \xi_{2}-L_{X_{2}} \xi_{1}+d_{A}\left(e_{1}, e_{2}\right)_{-}\right)
\end{aligned}
$$

其中

$$
\left(e_{1}, e_{2}\right)_{-}=\left(X_{1}+\xi_{1}, X_{2}+\xi_{2}\right)_{-}=\frac{1}{2}\left(\xi_{1}\left(X_{2}\right)-\xi_{2}\left(X_{1}\right)\right) .
$$

进一步, 在 Courant 代数胚 $E=A \oplus A^{*}$ 中, 子丛 $A$ 和 $A^{*}$ 都是 Dirac 结构.

定理 $16{ }^{[1]}$ 设 $E$ 是 Courant 代数胚, $L_{1}$ 和 $L_{2}$ 是 $E$ 中两个 Dirac 结构, 且相互横截, 即 $E=L_{1}$ $\oplus L_{2}$, 则 $L_{1}$ 与 $L_{2}$ 构成李双代数胚.

\subsection{Severa 类}

Severa ${ }^{[13,24]}$ 发现了一类称为正合 (exact) Courant 代数胚的重要构造. 下面简要介绍这种 Courant 代数胚及其分类.

定义 17 数域 $\mathbb{R}$ 上的 Courant 代数胚 $E$ 称为正合的, 如果下面的丛映射序列正合:

$$
0 \rightarrow T^{*} M \stackrel{\rho^{*}}{\rightarrow} E \stackrel{\rho}{\rightarrow} T M \rightarrow 0
$$

定义 18 设 $E$ 是上述正合 Courant 代数胚, 所谓一个 $E$ 的联络, 指这样的丛映射: $\gamma: T M \rightarrow E$, 满足 $\rho \circ \gamma=\mathrm{Id}_{T M}$ 和迷向性: $\langle\gamma(X), \gamma(Y)\rangle=0, \forall X, Y \in T M$.

定理 $19^{[13,24]}$ 设 $E$ 是上述正合 Courant 代数胚, 取定某个 $E$ 的联络 $\gamma$, 则

$$
\Omega(X, Y, Z):=\langle[\gamma(X), \gamma(Y)], \gamma(Z)\rangle, \quad \forall X, Y, Z \in \mathfrak{X}(M)
$$

定义了 $M$ 上的一个闭的 3- 形式. 且其对应的 3- 阶上同调类 $[\Omega] \in H_{\mathrm{deR}}^{3}(M)$, 不依赖于联络 $\gamma$ 的选 择. 称 $[\Omega]$ 为正合 Courant 代数胚的 Severa 同调类.

反过来, 给定某个闭的 3- 形式 $\Omega \in \Omega^{3}(M)$, 可以定义 $\Omega$ - 扭 Courant 代数胚 $C=T M \oplus T^{*} M$, 使 得 $C$ 上的内积和针仍然与原来标准的 Courant 代数胚一致, 但拥有新的 Courant 括号:

$$
\left[X_{1}+\xi_{1}, X_{2}+\xi_{2}\right]_{\omega}=\left[X_{1}+\xi_{1}, X_{2}+\xi_{2}\right]+\Omega\left(X_{1}, X_{2}, \cdot\right) .
$$

此时, $C$ 也是一个正合 Courant 代数胚, 且对应的 Severa 同调类就是 $[\Omega]$. 事实上, 这种对应关系即 Severa 给出的完全分类定理:

定理 $2^{[13,24]}$ 正合 Courant 代数胚的同构类一一对应于 $H_{\mathrm{deR}}^{3}(M)$.

\subsection{Dirac 结构的形变}

本小节介绍 Dirac 结构的形变以及用于刻画形变的 Maurer-Cartan 方程. 设 $E$ 是 Courant 代数 胚, 且其内积的符号是 $(n, n)$ 型的. 又设 $D \subset E$ 是一个 Dirac 结构. 可以任选一个秩为 $n$ 的子丛 $C \subset E$ 使得 $E \cong D \oplus C$ 且 $C$ 也是迷向的. 此时, 我们可以把 $C$ 等同于 $D^{*}$, 于是 $E \cong D \oplus D^{*}$.

首先简要介绍一种特殊情形, 就是 $C$ 可积: $[C, C] \subset C$, 此时 $C$ 也是 Dirac 结构. 这时, Dirac 结构 $D$ 的形变由某个满足下面条件的 $\omega \in \wedge^{2} C$ 控制:

$$
d_{D} \omega+[\omega, \omega]_{C}=0
$$


该方程也称作 Maurer-Cartan 方程. 详见文献 [1] 中的讨论.

下面进行更一般的讨论，也即 $C$ 未必是对合的情形. 设 $\Gamma(E)$ 的 Courant 括号为 $[\cdot, \cdot]_{E}$, 针为 $\rho_{E}: E \rightarrow T M$. 将这些运算限制到 $D^{*}$ 上, 得到 $\Gamma\left(D^{*}\right)$ 上的反对称括号 $[\cdot, \cdot]_{D^{*}}$ 和针映射 $\rho_{*}: D^{*} \rightarrow T M$ :

$$
\begin{aligned}
& {[\xi, \eta]_{D^{*}}=\operatorname{Pr}_{D^{*}}[\xi, \eta]_{E}, \quad \forall \xi, \eta \in \Gamma\left(D^{*}\right),} \\
& \rho_{*}=\left.\rho_{E}\right|_{D^{*}} .
\end{aligned}
$$

令 $\phi \in \Gamma\left(\wedge^{3} D\right)$ 定义为

$$
\phi(\xi, \eta, \zeta)=2\left\langle[\xi, \eta]_{E}, \zeta\right\rangle_{E}=2\left\langle\operatorname{Pr}_{D}[\xi, \eta]_{E}, \zeta\right\rangle_{E}, \quad \forall \xi, \eta, \zeta \in \Gamma\left(D^{*}\right),
$$

其中 $\langle\cdot, \cdot\rangle_{E}$ 是 $E$ 的内积. 容易验证上述 $\phi$ 的确是反对称的线性映射. 一般而言, $C \cong D^{*}$ 并不是 Dirac 结构. 换言之, $\phi$ 并不是平凡的, 所以, $\left(D^{*},[\cdot, \cdot]_{D^{*}}, \rho_{*}\right)$ 也不是李代数胚. 实际上, $\left(D, D^{*}\right)$ 构成拟李双 代数胚 (quasi-Lie bialgebroid) ${ }^{[39]}$.

令

$$
\lambda_{1}=d_{D}: \Gamma\left(\wedge^{k} D^{*}\right) \rightarrow \Gamma\left(\wedge^{k+1} D^{*}\right)
$$

表示来自李代数胚 $D$ 的 Chevalley-Eilenberg 微分算子. 下面定义二元运算:

$$
\lambda_{2}: \Gamma\left(\wedge^{k} D^{*}\right) \otimes \Gamma\left(\wedge^{l} D^{*}\right) \rightarrow \Gamma\left(\wedge^{k+l-1} D^{*}\right) .
$$

首先令

$$
\lambda_{2}(\xi, \eta)=[\xi, \eta]_{D^{*}}, \quad \lambda_{2}(\xi, f)=\rho_{*}(\xi)(f), \quad \forall \xi, \eta \in \Gamma\left(D^{*}\right), \quad f \in C^{\infty}(M),
$$

对其他类型的元素, 规定以 Leibniz 法则扩张上面在生成元上的运算即可.

类似地, 定义三元运算

$$
\lambda_{3}: \Gamma\left(\wedge^{k} D^{*}\right) \otimes \Gamma\left(\wedge^{l} D^{*}\right) \otimes \Gamma\left(\wedge^{r} D^{*}\right) \rightarrow \Gamma\left(\wedge^{k+l+r-3} D^{*}\right)
$$

为前述 $\phi$ 的通过 Leibniz 法则的扩张 (注意, 若 $\lambda_{3}$ 某个分量输入为 $M$ 上的 $\mathbb{K}$ - 值光滑函数, 则结果 为零).

下面的定理来自 Severa ${ }^{[24]}$ 与他人的通信, 可参见文献 [17], 类似的结论亦出现在文献 [40] 中.

命题 21 在上述假设下,

(1) 空间 $\Gamma\left(\wedge^{\bullet} D^{*}\right)$, 在赋予 $\lambda_{1} 、 \lambda_{2}$ 和 $\lambda_{3}$ 运算并令 $\lambda_{k}=0(k>3)$ 的设定下, 构成 $L$ - 无穷代数 (关 于 $L$ - 无穷代数, 又称强同伦李代数, 参见文献 [41]).

(2) 空间 $\Gamma\left(\wedge^{\bullet} D^{*}\right)$ 控制了 Dirac 结构 $D$ 的形变. 这意味着, 对于某个 $\omega \in \Gamma\left(\wedge^{2} D^{*}\right), D$ 沿着 $\omega$ 方 向的形变空间 $\left\{X+\omega^{b}(X) \mid X \in D\right\} \subset E$ 也是 Dirac 结构的充要条件是, $\omega$ 满足下面的 Maurer-Cartan 方程:

$$
\lambda_{1}(\omega)+\frac{1}{2} \lambda_{2}(\omega, \omega)+\frac{1}{6} \lambda_{3}(\omega, \omega, \omega)=0 .
$$

我们特别指出, 上述 $L$ - 无穷代数的构造, 虽然依赖于 $D$ 的补空间 $C$ 的选择, 但是, 其结构在 $L$ 无穷代数的同构意义下, 是规范不变的 (canonical) (参见文献 [24]). 


\section{Courant 代数胚的 Clifford 实现和导出括号}

\subsection{Clifford 代数与旋量表示}

设 $V$ 是某个 (数域 $\mathbb{K}$ 上的) $n$ - 维线性空间, 并带着非退化对称双线性型 $\langle\cdot, \cdot\rangle$. 所谓 $V$ 的 Clifford 代数, 记作 $\mathcal{C}(V)$, 指从张量代数 $\bigoplus_{k=0}^{n} V^{\otimes k}$ 按照关系

$$
x \otimes y+y \otimes x=2\langle x, y\rangle \quad(x, y \in V)
$$

生成等价关系后, 取该等价关系的商空间得到的代数.

自然地, $\mathcal{C}(V)$ 是一个结合的 $\mathbb{Z}_{2^{-}}$分次代数. 在最多差一个同构意义下, 存在 $\mathcal{C}(V)$ 唯一的不可约 模 $S$, 即所谓的 $\mathcal{C}(V)$ 的自旋表示 (参见文献 [42]). 此空间 $S$ 中的元素称为旋量 (spinor). 例如, 设 $W$ 是 $r$ - 维线性空间. 令 $V=W \oplus W^{*}$ 并取标准对称双线性型

$$
\left\langle u_{1}+\xi_{1}, u_{2}+\xi_{2}\right\rangle=\frac{1}{2}\left(\xi_{1}\left(u_{2}\right)+\xi_{2}\left(u_{1}\right)\right),
$$

其中 $u_{1}, u_{2} \in W, \xi_{1}, \xi_{2} \in W^{*}$. 在这种情形下, 令 $\mathcal{C}(V)$ 作用在 $S=\bigoplus_{k=0}^{r} \wedge^{k} W$ 上:

$$
u \cdot w=u \wedge w, \quad \xi \cdot w=\iota_{\xi} w,
$$

其中 $u \in W, \xi \in W^{*}, w \in S$. 此即自旋表示. 注意到 $S$ 是 $\mathbb{Z}$ - 分次, 也是 $\mathbb{Z}_{2^{-}}$分次的.

\subsection{Dirac 生成算子}

设 $\pi: E \rightarrow M$ 是某个 (数域 $\mathbb{K}$ 上的) 向量丛, 带有逐纤维定义的非退化对称双线性型 $\langle\cdot, \cdot\rangle$. 逐纤 维定义其对应的 Clifford 代数 $\mathcal{C}(E) \rightarrow M$. 又假设存在某个向量从 $S \rightarrow M$, 其逐点的纤维 $S_{m}(m \in M)$ 是相应的 Clifford 代数 $\mathcal{C}(E)_{m}$ 的旋量模. 进一步假设 $S$ 拥有 $\mathbb{Z}_{2^{-}}$分次: $S=S^{0} \oplus S^{1}$.

称一个 $\Gamma(S)$ 上的算子 $O$ 为偶的 (或者 0 次的), 如果 $O\left(S^{i}\right) \subset S^{i}$; 称之为奇的 (或者 1 次的), 如果 $O\left(S^{i}\right) \subset S^{i+1}$, 其中 $i \in \mathbb{Z}_{2}$. 例如, 若某个向量从 $E$ 可分解成 $A \oplus A^{*}$, 如前面例子所述, 则 $S=\wedge A$ 构成 $\mathcal{C}(E)$ 的旋量模. 此时, 乘以某个函数 $f \in C^{\infty}(M, \mathbb{K})$ 的运算是 $\Gamma(S)$ 的偶算子, 而某个截面 $e \in \Gamma(E)$ 在 $\Gamma(S)$ 上的作用是奇算子.

设 $O_{1}$ 和 $O_{2}$ 是两个算子, 分别具有次数 $d_{1}$ 和 $d_{2}$, 则定义它们的交换子为

$$
\left[O_{1}, O_{2}\right]=O_{1} \circ O_{2}-(-1)^{d_{1} d_{2}} O_{2} \circ O_{1} .
$$

定义 $22^{[21]}$ 称一个 $\Gamma(S)$ 上的奇算子 $D$ 为 $(E,\langle\cdot, \cdot\rangle)$ 的 Dirac 生成子, 如果 $D$ 满足下面的性质:

(1) 对任意 $f \in C^{\infty}(M, \mathbb{K}),[D, f] \in \Gamma(E)$. 这条性质意味着 $[D, f]$ 总是 $E$ 的某个截面的 Clifford 作用;

(2) 对任意 $e_{1}, e_{2} \in \Gamma(E)$, 有 $\left[\left[D, e_{1}\right], e_{2}\right] \in \Gamma(E)$;

(3) 算子 $D$ 的平方是某个 $M$ 上的函数的乘法: $D^{2} \in C^{\infty}(M, \mathbb{K})$.

在很多其他文献中提到的 “导出算子 (deriving operators)” 是一个更广泛的概念. 上述 Dirac 生 成算子是这种导出算子最早实例, 它具有下面的性质:

$$
\begin{aligned}
& {[[D, D], e]=0,} \\
& {\left[D,\left[e_{1}, e_{2}\right]\right]=\left[\left[D, e_{1}\right], e_{2}\right]-\left[e_{1},\left[D, e_{2}\right]\right],}
\end{aligned}
$$




$$
[[D, f], e]=[[D, e], f],
$$

其中 $f \in C^{\infty}(M, \mathbb{K}), e, e_{1}, e_{2} \in \Gamma(E)$.

我们以 Vinogradov 的例子 ${ }^{[12]}$ 说明上述概念. 设 $M$ 是光滑流形, 则 $E=T M \oplus T^{*} M$ 的截面在 $\Omega^{\bullet}(M)$ 上有如下定义的作用:

$$
(X+\xi) \cdot \mu=i_{X} \mu+\xi \wedge \mu,
$$

其中 $X \in \mathfrak{X}(M), \xi \in \Omega^{1}(M), \eta \in \Omega^{\bullet}(M)$. 如此定义的作用使得 $\Omega^{\bullet}(M)$ 成为 Clifford 代数 $\mathcal{C}(E)$ 的 Clifford 模, 其中 $E$ 的内积定义为 (2.3). 此时, 可以验证, de Rham 微分算子

$$
d: \Omega^{\bullet}(M) \rightarrow \Omega^{\bullet+1}(M)
$$

就是一个 Dirac 生成算子. 其诱导的 $\Gamma(E)$ 上的括号, 恰好就是我们前面提到的 Dorfman 括号 $(2.2)$. 事实上, Vinogradov 的构造与 Courant 和 Dorfman 的构造基本上是同一时间各自独立的研究工作中 的发现.

\section{3 李双代数胚与 Dirac 生成算子}

下面的定理说明 Dirac 生成算子可以导出 Courant 代数胚结构.

定理 $23^{[21]}$ 设 $D$ 是某个向量从 $\pi: E \rightarrow M$ 的 Dirac 生成算子, 则在 $E$ 上拥有诱导的 Courant 代数胚结构. 其针映射 $\rho: E \rightarrow T_{\mathbb{K}} M$ 定义为

$$
\rho(e) f=2\langle[D, f], e\rangle=[[D, f], e],
$$

其 Dorfman 括号定义为

$$
e_{1} \circ e_{2}=\left[\left[D, e_{1}\right], e_{2}\right] .
$$

下面介绍一个具体构造 Dirac 生成算子的例子, 详见文献 $[21,43]$. 设 $(A,[\cdot, \cdot], \rho)$ 与 $\left(A^{*},[\cdot, \cdot]_{*}, \rho_{*}\right)$ 构成一对互为对偶的李代数胚. 又设它们的秩都是 $n$, 底流形 $M$ 是 $m$ 维的. 考虑线从 $\wedge^{n} A^{*} \otimes \wedge^{m} T_{\mathbb{K}}^{*} M$, 它显然也是 $A^{*}$ - 模 (关于李代数胚及其模形式的内容可参见文献 [44]): 一个截面 $\alpha \in \Gamma\left(A^{*}\right)$ 作用在 $\Gamma\left(\wedge^{n} A^{*} \otimes \wedge^{m} T_{\mathbb{K}}^{*} M\right)$ 的定义为

$$
\alpha \cdot\left(\alpha_{1} \wedge \cdots \wedge \alpha_{n} \otimes \mu\right)=\sum_{i=1}^{n}\left(\alpha_{1} \wedge \cdots \wedge\left[\alpha, \alpha_{i}\right]_{*} \wedge \cdots \wedge \alpha_{n} \otimes \mu\right)+\alpha_{1} \wedge \cdots \wedge \alpha_{n} \otimes L_{\rho_{*}(\alpha)} \mu .
$$

若存在, 则该线从的平方根

$$
\mathscr{L}=\left(\wedge^{n} A^{*} \otimes \wedge^{m} T_{\mathbb{K}}^{*} M\right)^{\frac{1}{2}}
$$

也是李代数胚 $A^{*}$ - 模, 称作 Evens-Lu-Weinstein 模 ${ }^{[44]}$. 于是有相应的微分算子

$$
\breve{d}_{*}: \Gamma\left(\wedge^{k} A \otimes \mathscr{L}\right) \rightarrow \Gamma\left(\wedge^{k+1} A \otimes \mathscr{L}\right) .
$$

类似地, 若 $\left(\wedge^{n} A \otimes \wedge^{m} T_{\mathbb{K}}^{*} M\right)^{\frac{1}{2}}$ 存在, 则它也是李代数胚 $A$ - 模. 因此又有另一个微分算子

$$
\Gamma\left(\wedge^{k} A^{*} \otimes\left(\wedge^{n} A \otimes \wedge^{m} T_{\mathbb{K}}^{*} M\right)^{\frac{1}{2}}\right) \rightarrow \Gamma\left(\wedge^{k+1} A^{*} \otimes\left(\wedge^{n} A \otimes \wedge^{m} T_{\mathbb{K}}^{*} M\right)^{\frac{1}{2}}\right) .
$$

同时, 注意到下面的同构关系:

$$
\wedge^{k} A^{*} \cong \wedge^{n-k} A \otimes \wedge^{n} A^{*},
$$




$$
\begin{aligned}
& \wedge^{n} A^{*} \otimes\left(\wedge^{n} A \otimes \wedge^{m} T_{\mathbb{K}}^{*} M\right)^{\frac{1}{2}} \cong\left(\wedge^{n} A^{*} \otimes \wedge^{m} T_{\mathbb{K}}^{*} M\right)^{\frac{1}{2}}, \\
& \wedge^{k} A^{*} \otimes\left(\wedge^{n} A \otimes \wedge^{m} T_{\mathbb{K}}^{*} M\right)^{\frac{1}{2}} \cong \wedge^{n-k} A \otimes \wedge^{n} A^{*} \otimes\left(\wedge^{n} A \otimes \wedge^{m} T_{\mathbb{K}}^{*} M\right)^{\frac{1}{2}} \\
& \cong \wedge^{n-k} A \otimes\left(\wedge^{n} A^{*} \otimes \wedge^{m} T_{\mathbb{K}}^{*} M\right)^{\frac{1}{2}} .
\end{aligned}
$$

总之, 我们得到这样的微分算子

$$
\breve{\partial}: \Gamma\left(\wedge^{k} A \otimes \mathscr{L}\right) \rightarrow \Gamma\left(\wedge^{k-1} A \otimes \mathscr{L}\right) .
$$

下面的定理说明了如何判定 $\left(A, A^{*}\right)$ 构成李双代数胚, 以及相应的 Dirac 生成算子的构造.

定理 $24^{[43]}$ 互为对偶的李代数胚对 $\left(A, A^{*}\right)$ 是李双代数胚的充分必要条件是 $\breve{D}^{2} \in C^{\infty}(M, \mathbb{K})$. 换言之, 算子

$$
\breve{D}=\breve{d}_{*}+\breve{\partial}: \Gamma\left(\wedge^{\cdot} A \otimes \mathscr{L}\right) \rightarrow \Gamma\left(\wedge^{\prime} A \otimes \mathscr{L}\right)
$$

的平方是某个光滑函数 $\breve{f} \in C^{\infty}(M, \mathbb{K})$ 的乘法.

推论 25 [43] 互为对偶的李代数胚对 $\left(A, A^{*}\right)$ 是李双代数胚的充分必要条件是 $\breve{D}=\breve{d}_{*}+\breve{\partial}$ 构成 向量丛 $A \oplus A^{*}$ 的 Dirac 生成算子, 其中 $A \oplus A^{*}$ 拥有前面引入的标准内积 (3.8).

事实上, 由导出括号给出 Courant 代数胚的构造, 不止上述一种. 例如, Roytenberg ${ }^{[17]}$ 及 Roytenberg 和 Weinstein ${ }^{[18]}$ 利用分次 Poisson 流形的极小辛实现、同调 Hamilton 向量场和辛超流形定义了 Courant 代数胚的导出括号. 关于这两种导出括号的内在联系, 参见 Grutzman 等 ${ }^{[45}$ 近期的工作.

\section{5 广义复几何}

近年来, Courant 代数胚的理论得到很大发展. 例如, Hitchin 始创的广义复几何 [14] 就是完全建立 在 Courant 代数胚的理论之上. 本节简要介绍其基本理论框架.

\section{1 广义复结构}

首先, 在向量从 $T M \oplus T^{*} M$ 上, 回顾前面定义过的 Courant 括号 (2.2). 类似于复几何中的做法, 为了将广义复结构推广到流形上, 我们可以先定义一个广义近复结构, 即将广义复结构的线性理论逐 纤维定义到广义切丛上, 然后再附加上一个可积性条件.

定义 26 假设 $M$ 是 $2 n$ 维实流形. $M$ 上的广义近复结构由以下两个等价条件之一定义:

(1) $\mathcal{J}: T M \oplus T^{*} M \rightarrow T M \oplus T^{*} M$ 是从同态, 使得 $\mathcal{J}^{2}=-1$ 且 $\mathcal{J}^{*} \mathcal{J}=1$;

(2) $L$ 是 $T_{\mathbb{C}} M \oplus T_{\mathbb{C}}^{*} M$ 的一个极大迷向子丛, 且 $L \cap \bar{L}=\{0\}$, 其中 $T_{\mathbb{C}} M$ 和 $T_{\mathbb{C}}^{*} M$ 分别是 $T M$ 和 $T^{*} M$ 的复化从.

有了广义近复结构, 我们就可以考虑它的可积性. 类似于复几何中近复结构的挠率张量, 可以定 义广义近复结构的挠率张量:

$$
\mathcal{N}_{\mathcal{J}}(A, B)=[\mathcal{J} A, \mathcal{J} B]-\mathcal{J}[\mathcal{J} A, B]-\mathcal{J}[A, \mathcal{J} B]-[A, B], \quad \forall A, B \in T M \oplus T^{*} M .
$$

同样, 在将 $\mathcal{J}$ 和 Courant 括号复线性扩张到 $T_{\mathbb{C}} M \oplus T_{\mathbb{C}}^{*} M$ 上后, 易知挠率张量为零当且仅当

$$
[L, L] \subseteq L \quad \text { 或者 }[\bar{L}, \bar{L}] \subseteq \bar{L},
$$

其中 $L$ 是 $\mathcal{J}$ 在 $T_{\mathbb{C}} M \oplus T_{\mathbb{C}}^{*} M$ 上的特征 $i$ - 子丛, 其共轭 $\bar{L}$ 是 $\mathcal{J}$ 在 $T_{\mathbb{C}} M \oplus T_{\mathbb{C}}^{*} M$ 上的特征 $(-i)$ - 子丛. 
定义 27 称广义近复结构 $\mathcal{J}$ 是可积的, 如果它的特征 $i$ - 子丛 $L \subset T_{\mathbb{C}} M \oplus T_{\mathbb{C}}^{*} M$ 关于 Courant 括号是封闭的. 称可积的广义近复结构为广义复结构. 换句话说, 一个广义复结构就是一个复 Dirac 结构 $L$ 满足 $L \cap \bar{L}=\{0\}$.

我们注意到, 在该定义的条件下, $L$ 与 $\bar{L}$ 互为对偶丛, 且构成复李双代数胚.

定义 28 假设 $M$ 是一个光滑流形. 若 $M$ 上存在一个可积的广义近复结构 $\mathcal{J}$, 则称 $(M, \mathcal{J})$ 是 一个广义复流形.

例 29 如果 $\mathcal{J}: T M \oplus T^{*} M \rightarrow T M \oplus T^{*} M$ 形如

$$
\left(\begin{array}{cc}
J & 0 \\
0 & -J^{*}
\end{array}\right),
$$

其中 $J: T M \rightarrow T M$ 是逐点定义的丛映射, 则这样的 $\mathcal{J}$ 是广义复结构的充要条件就是 $J$ 为经典意义 下的流形 $M$ 上的复结构.

例 30 如果 $\mathcal{J}: T M \oplus T^{*} M \rightarrow T M \oplus T^{*} M$ 形如

$$
\left(\begin{array}{cc}
0 & -\omega^{-1} \\
\omega & 0
\end{array}\right),
$$

其中 $\omega: T M \rightarrow T^{*} M$ 是逐点定义的丛映射, 则这样的 $\mathcal{J}$ 是广义复结构的充要条件就是 $\omega$ 为经典意 义下的流形 $M$ 上的辛结构.

例 31 如果 $\mathcal{J}: T M \oplus T^{*} M \rightarrow T M \oplus T^{*} M$ 形如

$$
\left(\begin{array}{cc}
J & \pi \\
0 & -J^{*}
\end{array}\right),
$$

其中 $J: T M \rightarrow T M$ 和 $\pi: T^{*} M \rightarrow T M$ 是逐点定义的丛映射, 则这样的 $\mathcal{J}$ 是广义复结构的充要条件 就是 $J$ 为经典意义下的流形 $M$ 上的复结构, 且 $\pi$ 是一个全纯 Poisson 结构.

广义复流形是普通复流形和辛流形的结合体. 事实上, 目前普遍研究的广义复结构是额外带一个 扭项 (即某个闭的 3- 形式 $H \in Z^{3}(M)$ ) 的情形. 此时, 唯一变化的是 $T_{\mathbb{C}} M \oplus T_{\mathbb{C}}^{*} M$ 的 Courant 括号, 比原来多出来一个 $H$ - 扭部分:

$$
\left[X_{1}+\xi_{1}, X_{2}+\xi_{2}\right]_{H}=\left[X_{1}+\xi_{1}, X_{2}+\xi_{2}\right]+H\left(X_{1}, X_{2}, \cdot\right),
$$

其他结构不变. 仍旧按前述方式定义的广义复结构, 称为 $H$ - 扭广义复结构.

设 $(M, \mathcal{J})$ 是 $H$ - 扭广义复结构, 则 $\mathcal{J}$ 的 $i$ 特征子空间 $L$ 与 $(-i)$ 特征子空间 $\bar{L}$ 是互相横截的 ( $H$ - 扭) Dirac 结构, 仍然构成复李双代数胚. 反过来, 如果 $L$ 和 $\bar{L}$ 都是 Dirac 结构, 且 $L \cap \bar{L}=\{0\}$, 则它们唯一决定了某个 $H$ - 扭广义复结构. 也就是说, 互相横截且共轭的 Dirac 结构与 $H$ - 扭广义复 结构是一一对应关系.

\section{$5.2 \partial$ 与 $\bar{\partial}$ 算子}

考虑 $T_{\mathbb{C}} \oplus T_{\mathbb{C}}^{*}$ 生成的 Clifford 代数 $\mathcal{C}\left(T_{\mathbb{C}} \oplus T_{\mathbb{C}}^{*}\right)$, 对应的旋量从为

$$
\mathcal{M}:=\bigoplus_{i=0}^{2 n} \wedge^{i} T_{\mathbb{C}}^{*} .
$$


而 $\mathcal{C}\left(T_{\mathbb{C}} \oplus T_{\mathbb{C}}^{*}\right)$ 在 $\mathcal{M}$ 上的作用是由这样的关系生成的:

$$
(x+\eta) . \rho=\iota_{x} \rho+\eta \wedge \rho, \quad \forall \rho \in \mathcal{M} .
$$

此时, 可以找到一个称作纯旋量的线从 $N \subset \mathcal{M}$, 它满足下面的性质:

$$
L=\left\{l \in T_{\mathbb{C}} \oplus T_{\mathbb{C}}^{*} \mid l \cdot N=0\right\}, \quad \bar{L}=\left\{l \in T_{\mathbb{C}} \oplus T_{\mathbb{C}}^{*} \mid l \cdot \bar{N}=0\right\} .
$$

令 $N_{0}=N, N_{k}=\wedge^{k} \bar{L} \cdot N(k=0, \ldots, 2 n)$, 则有 $\bar{N}_{k}=N_{2 n-k}$. 特别地, $N_{2 n}=\bar{N}$ 恰是 $\bar{L}$ 的纯旋量 线从. 一个重要的结果是下面的直和分解 ${ }^{[15,16]}$ :

$$
\mathcal{M}=N_{0} \oplus N_{1} \oplus \cdots \oplus N_{2 n} .
$$

普通复流形上的 $\partial$ 和 $\bar{\partial}$ 算子, 也可以推广到 $H$ - 扭广义复流形的情形. 考虑算子

$$
d^{H}=d+H \wedge: \mathcal{M} \rightarrow \mathcal{M} .
$$

按照上述直和空间分解, 将微分算子 $d^{H}$ 分解成两部分: $d^{H}=\partial+\bar{\partial}$, 其中 $\partial: \Gamma\left(N_{\bullet}\right) \rightarrow \Gamma\left(N_{\bullet}-1\right)$, $\bar{\partial}: \Gamma\left(N_{\bullet}\right) \rightarrow \Gamma\left(N_{\bullet+1}\right)$. 换言之,

$$
\partial\left(n_{k}\right):=p r_{N_{k-1}}\left(d^{H} n_{k}\right), \quad \bar{\partial}\left(n_{k}\right):=p r_{N_{k+1}}\left(d^{H} n_{k}\right), \quad \forall n_{k} \in \Gamma\left(N_{k}\right) .
$$

下面简要介绍文献 [46] 中最重要的结果, 给出这两个算子的使用 Evens-Lu-Weinstein 模的刻画方 式. 换言之, $\partial$ 与 $\bar{\partial}$ 本质上就是复李双代数胚的 Chevalley-Eilenberg 微分算子.

定理 $32{ }^{[46]}$ 前述线丛 $\bar{N}=N_{2 n}$ 与 Evens-Lu-Weinstein 模 $\mathscr{L}=\left(\wedge^{2 n} \bar{L} \otimes \wedge^{2 n} T_{\mathbb{C}}^{*}\right)^{\frac{1}{2}}$ 同构. 进一步, 通过引入下列丛同构:

$$
\begin{aligned}
& I:\left(\wedge^{k} \bar{L}\right) \otimes N \rightarrow N_{k}, \quad W \otimes p \rightarrow W \cdot p, \quad \forall W \in \wedge^{k} \bar{L}, \quad p \in N, \\
& \bar{I}:\left(\wedge^{k} L\right) \otimes \bar{N} \rightarrow \bar{N}_{k}, \quad X \otimes \bar{p} \rightarrow X \cdot \bar{p}, \quad \forall X \in \wedge^{k} L, \quad p \in N,
\end{aligned}
$$

我们有下面的交换图表:

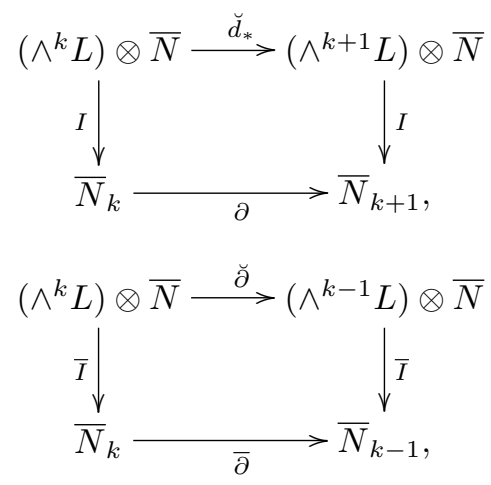

其中算子 $\breve{d}_{*}$ 和 $\breve{\partial}$ 是定义在前面的 (4.1) 和 (4.3).

这两个导算子与前面提到的 Dirac 生成算子有重要的联系. 事实上, 此时 $H$ - 扭 Courant 代数胚 $T_{\mathbb{C}} \oplus T_{\mathbb{C}}^{*}$ 的 Dirac 生成算子恰好就是

$$
d^{H}=\partial+\bar{\partial}=d+H \wedge .
$$


另外可以发现, 在上述同构解释下, 经过进一步计算有下面的显式表达:

$$
\begin{aligned}
& \bar{\partial}(W \cdot u)=\left(d_{L} W+e \wedge W\right) \cdot u, \quad \forall W \in \Gamma\left(\wedge^{k} \bar{L}\right), \\
& \partial(X \cdot \bar{u})=\left(d_{\bar{L}} X+\bar{e} \wedge X\right) \cdot \bar{u}, \quad \forall X \in \Gamma\left(\wedge^{i} L\right),
\end{aligned}
$$

其中 $u \in \Gamma(N)$ 是某个 (局部) 非平凡截面, $e \in \Gamma(\bar{L})$ (即 $L$ 的模闭链 (modular cocycle)) 使得 $\bar{\partial} u=e . u$.

关于这广义复结构的两个算子更多的深入研究和结果, 参见文献 [46]. 另外, 与此紧密相关的, Stiénon ${ }^{[47]}$ 发现了经典辛几何中的 Moser 引理在广义复几何中的非平凡推广.

\section{6 正则 Courant 代数胚}

本节简要介绍正则 Courant 代数胚的结构和分类, 详细内容参见文献 [28].

在数域 $\mathbb{K}$ 为 $\mathbb{R}$ 的情形, 如前定义的 Courant 代数胚 $E$ 称作是正则的, 如果其中针映射 $\rho$ 是正则 的, 即 $\operatorname{rank}(\rho(E))$ 为常值. 正则 Courant 代数胚蕴含了一系列重要的空间信息: 如下图所示, 共有 4 条 从左到右相互交错的正合列. 特别地, 居中的 $\mathcal{G}$ 是一个二次李代数从 (quadratic Lie algebra bundle).

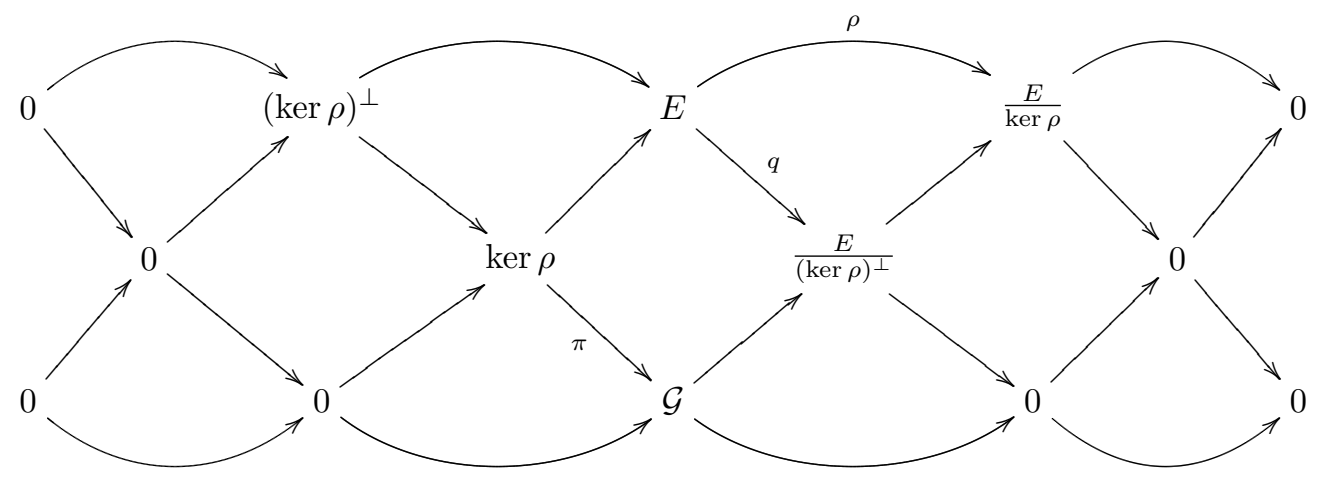

这里的 $\mathcal{G}:=\operatorname{ker} \rho /(\operatorname{ker} \rho)^{\perp}$. 符号 $\pi$ ( 以及 $q$ ) 表示投影映射 $\operatorname{ker} \rho \rightarrow \mathcal{G}$ (以及 $E \rightarrow \mathcal{A}_{E}=E /(\operatorname{ker} \rho)^{\perp}$ ).

在研究正则 Courant 代数胚之前, Vaisman ${ }^{[48]}$ 和 Bressler [49] 分别深入研究了可递 (transitive) Courant 代数胚, 即 $\rho(E)=T M$ 的情形, 获得很多重要的进展, 发掘出很多深刻的性质和应用.

我们知道, 正则 Courant 代数胚的特例就是可递 Courant 代数胚, 后者的特例就是正合 Courant 代数胚. 基于以上这些进展, 文献 [28] 的主要目的就是给出最一般的正则 Courant 代数胚的分类定理. 另外, 我们需要探索与正则 Courant 代数胚有关的几个重要的同调群之间的联系.

下面简要介绍文献 [28] 中的主要研究成果. 首先, 对任何正则 Courant 代数胚 $E$, 在其 3 阶初级 同调群 (naive cohomology) 中, 蕴含一个自然的元素 $C_{E} \in H_{\text {naive }}^{3}(E)$, 命名为 $E$ 的特征类.

该特征类在极端的情形, 还原为 Severa 特征类或者 Cartan 形式. 但是, $C_{E}$ 并不能完全分类这样 的正则 Courant 代数胚. 事实上, 真正的分类定理的完整表述要复杂得多. 我们将其分解为下列两个 定理:

定理 $33^{[28]}$ 存在从全体正则 Courant 代数胚到全体第一 Pontryagin 类 (简记为 FPC) 为零的 二次李代数胚 (quadratic Lie algebroid) 的自然函子, 称作 ample 函子, 记作 $\mathcal{J}$, 其定义为

$$
\mathcal{J} E=E /(\operatorname{ker} \rho)^{\perp} .
$$


该函子满足关系

$$
H_{\text {naive }}^{k}(E)=H^{k}(\mathcal{J} E) .
$$

定理 $34^{[28]}$ 对任意 $\mathrm{FPC}$ 为零的二次李代数胚 $A$ 的同构类, 其在 ample 函子下的逆像 $\mathcal{J}^{-1}[A]$ 可以参数化为集合 $\rho_{A}^{*} H^{3}(F) / I$, 其中 $\rho_{A}$ 是 $A$ 的针映射, $F=\operatorname{ker} \rho_{A}, I$ 是 $\rho_{A}^{*} H^{3}(F)$ 的某个 Abel 子群.

对于正则 Courant 代数胚 $E$, 记其对应的二次李代数胚为 $A=\mathcal{J} E$, 则前述 Courant 代数胚 $E$ 的 特征类可以视作 $C_{E} \in H^{3}(A)$. 它还满足我们称之为凝聚的 (coherent) 性质, 即在集合

$$
\left\{(A, C) \mid A \text { 是 } \mathrm{FPC} \text { 为零的二次李代数胚, 且 } C \in H^{3}(A) \text { 是凝聚的 }\right\}
$$

中引入一种等价关系 “ ” (详见文献 [28]), 则有下面的定理:

定理 $35^{[28]}$ 存在正则 Courant 代数胚同构类的集合与 $\{(A, C)\}$ 的等价类集合之间的一一对应.

以上就是我们给出的正则 Courant 代数胚完全分类定理. 更深入的关于正则 Courant 代数胚及其 三阶初级同调特征类的研究结果, 参见文献 [28]. 应用这些方法和结论, 我们进一步发掘了许多崭新且 极为精细的性质, 以及很多具体的实例应用. 我们注意到该结果彻底完整地分析清楚了正则 Courant 代数胚的结构问题, 并给出了正则 Courant 代数胚的局部构造, 得到了 Poisson 结构的 Weinstein 局部 分裂定理在 Courant 代数胚情形的表达形式. 最重要的是, 发现了决定正则 Courant 代数胚的同构分 类的要素是一个初级三阶上同调类, 它是正则条件下把 Cartan 三形式与 Severa 特征类统一的构造. 由此建立了 Courant 代数胚的第二示性类 (secondary characteristic class) 理论. 这在很大程度上给出 了进一步寻找更高层的特征类的可能性及构造方式.

至此, 我们简要介绍了关于 Courant 代数胚的研究背景和历史发展, 特别介绍了 Courant 代数胚 和一些重要的数学对象的关系和研究进展. 目前, 我们正在密切关注与 Courant 代数胚有关的课题的 最新发展动态. 作者也正在从事几项重要的问题研究及 Courant 代数胚的其他更深入的应用. 例如, 与 广义复几何密切相关的扩展 Poisson 结构 [50] 和全纯 Poisson 结构 [51] 等. 我们还基于近期关于 Atiyah 同调类的重要结果 (参见文献 [52]), 设想在广义复几何框架下的 Atyiah 同调类也应该存在, 而且有更 丰富的结构. 还有基于已有的研究 Dirac 结构的结果 (参见文献 [53]), 正在深入研究广义接触结构等 相关问题. 我们期望在不远的将来与读者分享这些相关最新的研究结果和论文.

致谢 郎红蕾和李彦鹏博士对本文修改提出很多有益的建议, 在此表示感谢.

\section{参考文献}

1 Liu Z J, Weinstein A, Xu P. Manin triples for Lie bialgebroids. J Differential Geom, 1997, 45: 547-574

2 Merker J. On the geometric structure of Hamiltonian systems with ports. J Nonlinear Sci, 2009, 19: 717-738

3 Roytenberg D. AKSZ-BV formalism and Courant algebroid-induced topological field theories. Lett Math Phys, 2007, 79: $143-159$

4 Grana M, Minasian R, Petrini M, et al. T-duality, generalized geometry and non-geometric backgrounds. J High Energy Phys, 2009, 2009: 075

5 Grabowska K, Grabowski J. Dirac algebroids in Lagrangian and Hamiltonian mechanics. J Geom Phys, 2011, 61: 2233-2253

6 Yoshimura H, Marsden J E. Dirac structures in Lagrangian mechanics, I: Implicit Lagrangian systems. J Geom Phys, 2006, 57: 133-156

7 Weinstein A. Coisotropic calculus and Poisson groupoids. J Math Soc Japan, 1988, 40: 705-727

8 Mackenzie K C H, Xu P. Lie bialgebroids and Poisson groupoids. Duke Math J, 1994, 73: 415-452

9 Drinfel'd V. Quantum groups. In: Proceedings ICM Berkeley. Providence: Amer Math Soc, 1987, 789-820 
10 Drinfel'd V G. Hamiltonian structures on Lie groups, Lie bialgebras and the geometric meaning of classical Yang-Baxter equations. Dokl Akad Nauk SSSR, 1983, 268: 285-287

11 Courant T J. Dirac manifolds. Trans Amer Math Soc, 1990, 319: 631-661

12 Cabras A, Vinogradov A M. Extensions of the Poisson bracket to differential forms and multi-vector fields. J Geom Phys, 1992, 9: 75-100

13 Severa P, Weinstein A. Poisson geometry with a 3-form background. Progr Theoret Phys Suppl, 2001, 144: 145-154

14 Hitchin N. Generalized Calabi-Yau manifolds. Q J Math, 2003, 54: 281-308

15 Gualtieri M. Generalized complex geometry. Ann of Math (2), 2011, 174: 75-123

16 Cavalcanti G R, Gualtieri M. Generalized complex geometry and T-duality. In: A Celebration of the Mathematical Legacy of Raoul Bott, vol. 50. Providence: Amer Math Soc, 2010, 341-365

17 Roytenberg D. Courant algebroids, derived brackets and even symplectic supermanifolds. PhD Thesis. Berkekey: University of California Berkeley, 1999

18 Roytenberg D, Weinstein A. Courant algebroids and strongly homotopy Lie algebras. Lett Math Phys, 1998, 46: 81-93

19 Loday J L. Une version non commutative des algebres de Lie: Les algebres de Leibniz. Res Cooperat Program, 1992, 44: $127-151$

20 Dorfman I Y. Dirac structures of integrable evolution equations. Phys Lett A, 1987, 125: 240-246

21 Alekseev A, Xu P. Derived brackets and Courant algebroids. Unpublished Manuscript. Http://www.math.psu.edu/ ping/anton-final.pdf, 2001

22 Kosmann-Schwarzbach Y. Derived brackets. Lett Math Phys, 2004, 69: 61-87

23 Hull C M. Generalised geometry for M-theory. J High Energy Phys, 2007, 2007: 079

24 Severa P. Letters to Alan Weinstein about Courant algebroids. ArXiv:1707.00265, 2017

25 Stiénon M, Xu P. Modular classes of loday algebroids. C R Math Acad Sci Paris, 2008, 346: 193-198

26 Ginot G, Grutzmann M. Cohomology of Courant algebroids with split base. J Symplectic Geom, 2009, 7: 311-335

27 Weinstein A. 群胚: 内部与外部对称的统一. 数学译林, 2002, 1: 15-26

28 Chen Z, Stiénon M, Xu P. On regular Courant algebroids. J Symplectic Geom, 2013, 11: 1-24

29 Liu Z J, Sheng Y H, Xu X M. The Pontryagin class for pre-Courant algebroids. J Geom Phys, 2016, 104: 148-162

30 Chen Z, Liu Z J, Sheng Y H. E-Courant algebroids. Int Math Res Not IMRN, 2010, 2010: 4334-4376

31 Li-Bland D, Severa P. Integration of exact Courant algebroids. Electron Res Announc Math Sci, 2012, 19: 58-76

32 Mehta R, Tang X. From double Lie groupoids to local Lie 2-groupoids. Bull Braz Math Soc (NS), 2011, 42: 651-681

33 Sheng Y H, Zhu C C. Higher extensions of Lie algebroids. Commun Contemp Math, 2017, 19: 1650034

34 Kosmann-Schwarzbach Y. Courant algebroids. A short history. SIGMA Symmetry Integrability Geom Methods Appl, 2013, 9: 8 papers

35 Pradines J. Troisième théorème de Lie sur les groupoïdes différentiables. C R Math Acad Sci Paris, 1968, 267: 21-23

36 Ehresmann C. Oeuvres complètes et commentées. Supplements to Cahiers de topologie et géométrie différentielle (edited and commented by Andrée Charles Ehresmann). Http://ehres.pagesperso-orange.fr/Cahiers/Ctgdc.htm, 19801984

37 Mackenzie K C H. General Theory of Lie Groupoids and Lie Algebroids. London Mathematical Society Lecture Note Series, vol. 213. Cambridge: Cambridge University Press, 2005

38 Xu P. Gerstenhaber algebras and BV-algebras in Poisson geometry. Comm Math Phys, 1999, 200: 545-560

39 Roytenberg D. Quasi-Lie bialgebroids and twisted Poisson manifolds. Lett Math Phys, 2002, 61: 123-137

40 Keller F, Waldmann S. Formal deformations of Dirac structures. J Geom Phys, 2007, 57: 1015-1036

41 Lada T, Stasheff J. Introduction to SH Lie algebras for physicists. Internat J Theoret Phys, 2993, 32: 1087-1103

42 Chevalley C. The Algebraic Theory of Spinors and Clifford Algebras: Collected Works, vol. 2. Berlin: Springer-Verlag, 1997

43 Chen Z, Stiénon M. Dirac generating operators and Manin triples. J Lond Math Soc (2), 2009, 79: 399-421

44 Evens S, Lu J H, Weinstein A. Transverse measures, the modular class and a cohomology pairing for Lie algebroids. Quart J Math Oxford Ser (2), 1999, 50: 417-436

45 Grutzmann M, Michel J P, Xu P. Weyl quantization of degree 2 symplectic graded manifolds. ArXiv:1410.3346, 2014

46 Chen Z. The operators $\partial$ and $\bar{\partial}$ of a generalized complex structure. Pacific J Math, 2009, 242: 53-69

47 Stiénon M. Moser lemma in generalized complex geometry. Trans Amer Math Soc, 2010, 362: 5107-5123

48 Vaisman I. Transitive Courant algebroids. Int J Math Math Sci, 2005, 2005: 1737-1758

49 Bressler P. The first Pontryagin class. Compos Math, 2007, 143: 1127-1163

50 Chen Z, Stiénon M, Xu P. Geometry of Maurer-Cartan elements on complex manifolds. Comm Math Phys, 2010, 297: 169-187 
51 Chen Z, Fino A, Poon Y S. Holomorphic Poisson structure and its cohomology on nilmanifolds. Differential Geom Appl, 2016, 44: 144-160

52 Chen Z, Stiénon M, Xu P. From Atiyah classes to homotopy Leibniz algebras. Comm Math Phys, 2016, 341: 309-349

53 Chen Z, Liu Z J, Sheng Y H. Dirac structures of omni-Lie algebroids. Internat J Math, 2011, 22: 1163-1185

\section{Courant algebroids and generalized complex geometry}

\section{CHEN Zhuo, LIU ZhangJu \& XU Ping}

Abstract In this survey, we recall the background and major developments in Courant algebroids and their applications. We mainly recall the origin and motivation of the definition of Courant algebroids. A brief introduction to the theories of Dirac structures, Lie bialgebroids, the Clifford construction, spinor representation, generalized complex geometry and regular Courant algebroids are given.

Keywords Courant algebroid, Dirac structure, Lie bialgebroid, generalized complex geometry $\mathrm{MSC}(2010) \quad 53 \mathrm{C} 15,53 \mathrm{D} 17,57 \mathrm{R} 15,58 \mathrm{~A} 10,15 \mathrm{~A} 66,17 \mathrm{~B} 63,17 \mathrm{~B} 66$

doi: $10.1360 /$ N012017-00159 